\title{
Linear Pharmacokinetic Models and Vanishing Exponential Terms: Implications in Pharmacokinetics
}

\author{
John G. Wagner ${ }^{1,2}$ \\ Received Jan. 8, 1976-Final Apr. 9, 1976
}

\begin{abstract}
A series of models more closely related than classical models to known facts about drug absorption and disposition are presented. It is shown that mathematically such models require the same number of exponential terms in the equation describing the plasma concentration following intravenous administration as in that describing the plasma concentration following oral administration. However, it is also shown that one or two of the exponential terms of the intravenous, and sometimes the oral, equation often are relatively unimportant and appear to vanish on stripping or fitting of data. This phenomenon leads to ambiguity concerning which model to assign to one or more sets of data. The number of potential models in a given situation has now been greatly increased. It is also shown that if data obey these models then neither the Wagner-Nelson nor the Loo-Riegelman method provides estimates of absorption rate constants when the $A_{t} / V_{p}, t$ data are analyzed by conventional methods.
\end{abstract}

KEY WORDS: inseparable input and disposition portions of models; vanishing exponential terms; drug absorption and disposition models; absorption rate constants; model-independent pharmacokinetics; polyexponential computer fitting.

\section{INTRODUCTION}

One method of classifying linear pharmacokinetic models is shown in Figs. 1 and 2. Figure 1 shows some classical linear pharmacokinetic models in which transfer from input to disposition compartments occurs in only one direction. This leads to a separation of input and disposition functions, and the Laplace transform methods of Benet (1) and Vaughan and Trainor (2)

Supported in part by Public Health Service Grant 5P11 GM 15559.

${ }^{1}$ College of Pharmacy and Upjohn Center for Clinical Pharmacology, The University of Michigan, Ann Arbor, Michigan 48109.

${ }^{2}$ Address reprint requests to Dr. John G. Wagner, Upjohn Center for Clinical Pharmacology, University of Michigan Medical Center, Ann Arbor, Michigan 48109. 
rely on such a "separation." Figure 2 shows some nonclassical linear pharmacokinetic models in which there are reversible transfers between the terminal input compartment and one of the disposition compartments; the models shown also have reversible transfers between all input compartments, but these are not essential to produce the effects to be described. In models I-A and II-A, compartment 1 represents small intestinal contents as the absorption site; compartment 2 represents not only plasma but also tissues which bind the drug, such that the drug in tissue is in equilibrium with free drug in plasma water and there is a constant tissue concentration/free drug concentration in plasma water ratio (3); compartment 3 of model II-A represents tissues and/or other fluids of distribution and is analogous to the usual second compartment of the classical "two-compartment open model." In models I-B, I-C, II-B, and II-C, compartment 1 represents the stomach contents, and compartment 2 represents the small intestinal contents; compartment 3 in these models is analogous to compartment 2 in models I-A and II-A, and compartment 4 in these models is analogous to compartment 3 in model II-A.

Thus $k_{12}$ for models I-A and II-A and $k_{23}$ for models I-B, I-C, II-B, and II-C are intestinal absorption rate constants representing transfer of drug

"One Compartment open Disposition Models" with Input

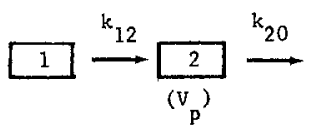

"Two Compartment Open Disposition Moeels" with Input
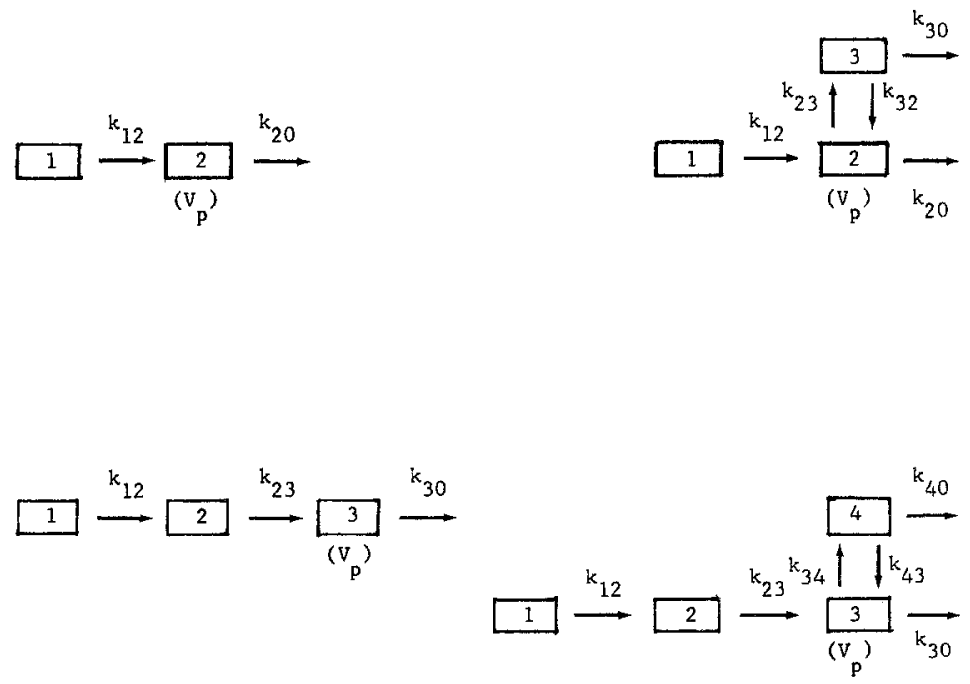

Fig. 1. Classical linear pharmacokinetic models. 
Analogues of "One Compartment Open

Disposition Models" with Input

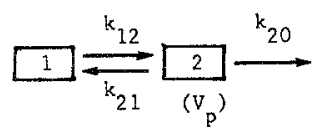

$\mathrm{I}-\mathrm{A}$

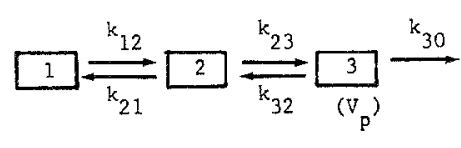

$I-B$

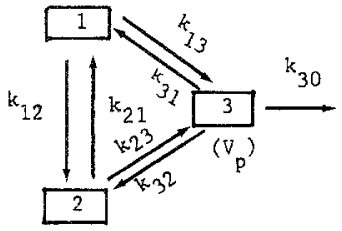

I-C
Analogues of "Two Compartment Open Disposition Models" with Input

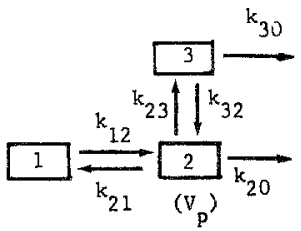

II-A

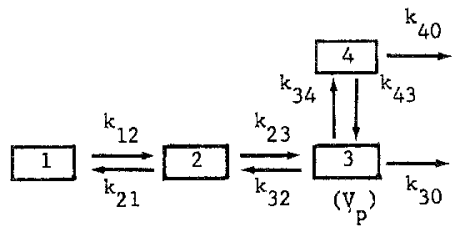

II-B

Fig. 2. Nonclassical linear pharmacokinetic models.

from the intestinal fluids to the circulation. The $k_{21}$ for models I-A and II-A and the $k_{32}$ for models I-B, I-C, II-B, and II-C represent transfer of drug back from the circulation to the intestinal fluids (as, for example, by intestinal secretion). If models I-B, I-C, II-B, and II-C apply in the real world, one would usually expect $k_{21}=0$, since it would seemingly require regurgitation to get drug from the intestinal contents back to stomach contents. However, even if $k_{21}=0$ in these models, the net result is the same and the conclusions are the same; only the meanings of the $E_{i}$ 's and $\lambda_{i}$ 's change. 
The $k_{13}$ of models I-C and II-C represent gastric absorption rate constants. The $k_{31}$ of these models represent transfer of drug from the circulation to stomach contents (i.e., gastric secretion), such as in $p \mathrm{H}$ partition (4).

As in the classical models of Fig. 1, all rate constants in the models of Fig. 2 are first order. Models of both Figs. 1 and 2 are more rigorously applicable to real-life situations when oral dosing (i.e., placing the drug in compartment 1) with solutions of a drug. When oral dosing involves solid dosage forms, dissolution as well as absorption processes are involved and the situation is much more complicated. However, experience has shown that the equations derived from models such as shown in Fig. 1 have been successfully utilized in the fitting of whole blood (plasma or serum) concentration-time data following oral administration of solid dosage forms; there is no reason to believe that the same would not hold for the models of Fig. 2. For the nonclassical models (Fig. 2), bolus intravenous administration is simulated by putting the dose at zero time into compartment 2 of models I-A and II-A and into compartment 3 of models I-B, I-C, II-B, and II-C.

The purpose of this article is not to suggest that we should try to fit real data to equations appropriate for the schematic models shown in Fig. 2; in most cases, there would be insufficient information on blood concentrationtime data alone to be able to do so. Rather, the purpose is to show that most of the time there is ambiguity concerning which model to assign to one or more sets of data. The models of Fig. 2 lead to cyclic phenomena with respect to the drug-i.e., drug at an absorption site is absorbed and reaches the circulation, but some drug is returned from the circulation to the absorption site or sites. The occurrence of processes contributing to such cyclic phenomena will be discussed first. Then the magnitude and possible importance of these phenomena with some drugs will be more explicitly discussed.

\section{Occurrence of Processes}

It is well known that (a) some drugs are absorbed from the stomach (5-11) as well as from the intestine; (b) acidic and basic drugs are secreted into gastric juice following intravenous (and oral) administration (4,5); (c) many drugs are secreted into the intestinal lumen $(12,13)$; (d) many drugs are secreted into saliva (14a, 15a), the saliva is swallowed, and the drug is available for both gastric and intestinal absorption; and (e) many drugs enter into an enterohepatic cycle in which the drug leaves the circulation and is concentrated in bile, the bile enters the intestinal lumen, and the drug is reabsorbed. The classical linear pharmacokinetic models of Fig. 1 ignore these facts. 


\section{Magnitude of the Effects}

One might be misled by attempting to predict the relative importance of gastric and intestinal absorption on the basis of the small percentage of the cardiac output delivered to the stomach relative to that delivered to the intestinal mucosa, the relative blood flow rates, and the relative absorbing surface areas of the stomach and small intestine. A most important factor is residence time in the stomach, which of course differs appreciably under fasting and nonfasting conditions, and may be altered by either the drug itself or a coadministered drug.

Table I lists gastric and intestinal absorption rate constants of 13 barbiturates in the rat reported by Kakemi et al. $(11,16)$. The fifth column of Table I lists the gastric absorption rate constant as a percentage of the intestinal absorption rate constant; these values range from 2.99 to $30.9 \%$. There is a trend for these values to increase with increase in the logarithm of the chloroform $/ p \mathrm{H} 1.1$ aqueous buffer partition coefficient, which is shown in the last column of the table. The important point to be gained from these data is that one cannot always assume that the contribution of gastric absorption is very small; the contribution is different even for each drug in an analogue series, and with some members of such a series reaches a magnitude such that the contribution of gastric absorption should not be ignored pharmacokinetically.

Hogben et al. (5) reported that $40 \%$ of ethanol was absorbed from the stomach of man in a 20 -min period when gastric emptying was not impeded. Cooke and Birchall (6) administered 95 test meals to seven subjects. The test meals consisted of $350 \mathrm{ml}$ of water containing phenol red as a marker, $35 \mathrm{~g}$ of glucose to delay gastric emptying and ensure a relatively constant volume remaining in the stomach at $30 \mathrm{~min}$, and various concentrations of ethanol. The gastric contents were aspirated after $30 \mathrm{~min}$; then another $250 \mathrm{ml}$ of water was swallowed and the contents were aspirated to recover any residual gastric contents. From their data on seven subjects each given four different doses of ethanol, the authors calculated that the mean percentages of the doses of ethanol which were absorbed from the stomach were $30.7,27.3$, 27.8 , and $28.1 \%$ for concentrations of ethanol in the test meals of $7.9,15.1$, 29.3 , and $55.8 \%$, respectively.

The $p \mathrm{H}$-partition hypothesis (4) assumes that only un-ionized molecules are absorbed. However, there is considerable evidence that drug ions are also absorbed. For example, Fiese and Perrin $(17,18)$ studied absorption of dextromethorphan, with reported $p \mathrm{~K}_{\mathrm{a}}$ of 7.97 or 8.25 , at $p \mathrm{H} 2.0$ from rat stomach. They reported (17) that $25.3,29.7,28.7,27.8$, and $24.0 \%$ of the dose was absorbed following doses of $0.15,0.30,0.60,0.90$, and $1.5 \mathrm{mg}$ of dextromethorphan, respectively. In a later article (18), they 


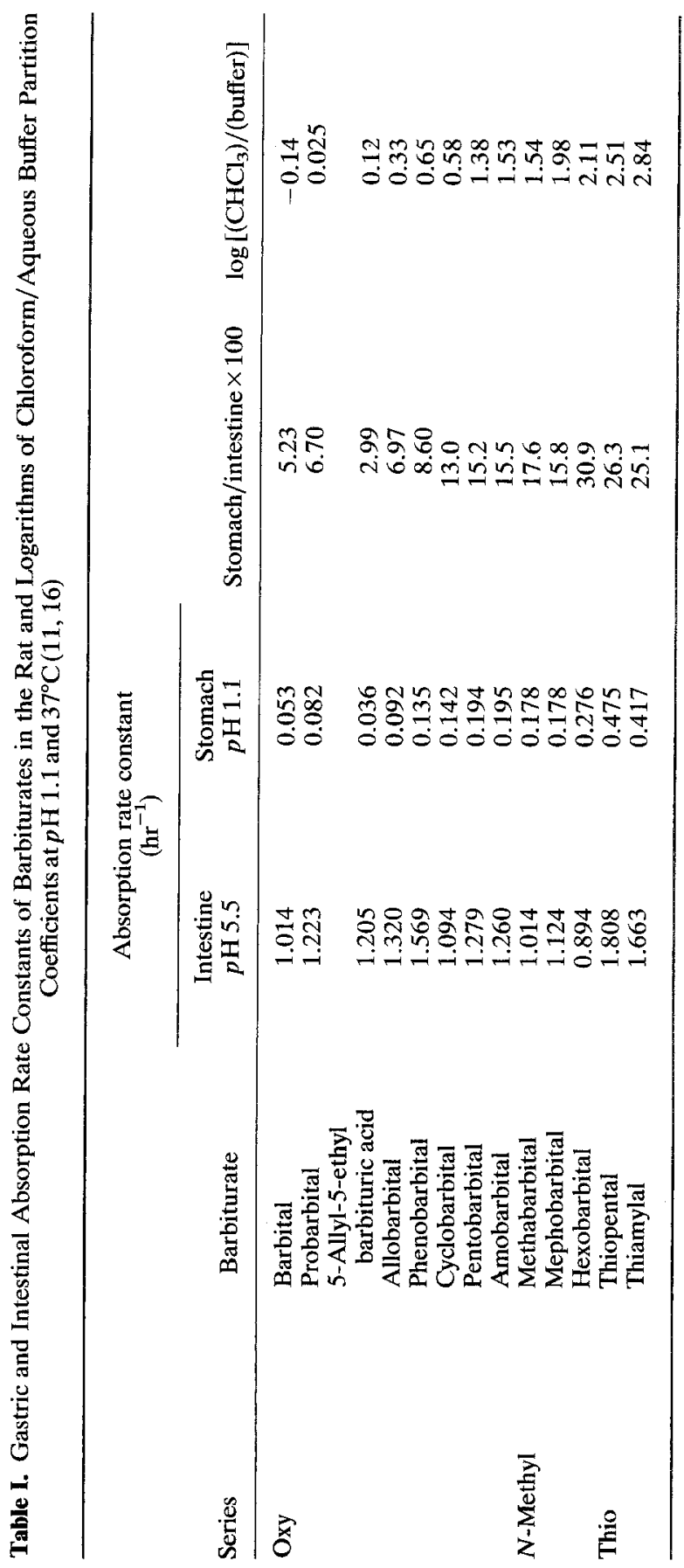


speculated that the protonated dextromethorphan concentrates on the stomach wall and that this surface concentration accounts for the absorption. Martin (19) also discussed the accumulation of drug anions in gastric mucosal cells and showed that such accumulation would be considerably greater with the stronger acids.

Hellström et al. (7) reported that an average of $11 \%$ of potassium ${ }^{35} \mathrm{~S}$-phenoxymethyl penicillin in nine subjects was absorbed from the stomach of man following oral administration of $15 \mu \mathrm{C}_{\mathrm{i}}(3 \mathrm{~g})$ of the salt in $50 \mathrm{ml}$ of water containing $5 \mathrm{~g}$ of polyethylene glycol as a marker.

There is also the danger of extrapolating in situ absorption data. Taylor and Grundy (20) measured absorption of practolol, propranolol, and salicyclic acid by both in situ and in vivo methods in the rat. The most striking difference was with practolol, where absorption was not measurable at all by the in situ method, but the absorption half-life was $10 \mathrm{~min}$ by the in vivo method. With propranolol, absorption half-times were 15 and 5 min by in situ and in vivo methods, respectively; for salicyclic acid, they were 8 and 5 min.

In pharmacokinetics we have usually only considered the unidirectional movement of drugs across the intestinal membrane and have ignored much of the literature $(12,13,21,22 \mathrm{a})$ indicating movement in both directions. In man, the minimum rate of water absorption in the small intestine averages $200-400 \mathrm{ml} / \mathrm{hr}$ and the net flow of water across the intestinal membrane is equal to the difference between the unidirectional flux from intestinal lumen to interstitial fluid (and eventually into blood and lymph) and the opposite flux from the interstitial fluid to intestinal lumen; actually, net water absorption is the difference between two very rapid, oppositely oriented fluxes, and a small change in either individual flux can cause a large change in net flow (22a). A similar situation exists with respect to ions, such as $\mathrm{Na}^{+}$, $\mathrm{Cl}^{-}$, and $\mathrm{SO}_{4}{ }^{2-}$, and with sugars (22a). In the rat, absorption of xylose is proportional to water absorbed (22a), and the same has been reported for $\mathrm{Na}^{+}$in the rat (21). Thus there is often a linkage between water and solute movement in the intestinal tract (21). About 8.5 liters of fluid enters the upper small intestine of man per day ( 7 liters of digestive juices and 1.5 liters of oral intake) (21). Material emptied from the stomach of man is mixed with about $1500 \mathrm{ml}$ of fluid in the upper small intestine (22a). On the other side of the membrane, an adult circulates about $3000 \mathrm{ml}$ of plasma in about half a minute (14b). Hence with respect to movement of the diffusible free (unbound) drug species it is doubtful that the relative volumes can be ignored. This is illustrated with a simulation example with model II-A later in the experimental section.

Winne (23) discussed how intestinal blood flow may affect absorption and elaborated two types of models which incorporated the intestinal lumen, 
the interstitial space, and the streaming blood; movement across the intestinal epithelium was assumed to occur in both directions, consistent with that above. However, Williams et al. (24) showed experimentally that exercise in the heat, which diminishes splanchnic blood flow, markedly reduced absorption of the actively absorbed sugar 3- $O$-methyl-D-glucose but did not affect absorption of the passively absorbed sugar $\mathrm{D}$-xylose in man.

Crouthamel and Abolin (13) reported that after intravenous administration of tetracycline in the rat, duodenal concentrations were equal to serum concentrations in $1 \mathrm{hr}$ with normal bile flow and in $2 \mathrm{hr}$ with the bile duct ligated. They concluded that the return of tetracycline from blood to the intestine has a significant effect on tetracycline pharmacokinetics. Holland and Quay (25) showed that concentrations of erythromycin base in the intestinal lumen of the rabbit considerably exceeded concentrations in plasma from $80 \mathrm{~min}$ onward after initiation of intravenous infusions of erythromycin base. Preloading of the intestine with 5 and 20 times the plasma concentration did not diminish the intestinal secretion of erythromycin, suggesting that the secretion was active. Lauterbach (12) summarized his own and others' investigations on intestinal secretion. He reported that cardiac glycosides, quaternary ammonium compounds, and strongly acidic drugs have greater fluxes from serosal to mucosal sides than from mucosal to serosal sides. The apparently active secretory mechanisms for these classes of compounds were substantiated in vivo. After intravenous administration, cardiac glycosides and quaternary ammonium compounds were concentrated in the intestinal lumen well above the serum level in the guinea pig and the rat. The establishment of an equilibrium between the concentrations in the blood and the intestinal lumen was demonstrated to be the cause of the previously observed standstill in the absorption of quaternary ammonium compounds despite considerable amounts of unabsorbed drug. These results reveal the intestine as the third excretory organ besides the liver and the kidney (12).

The salivary glands of man secrete 1-2 liters of saliva per day (22b). The free drug concentration in plasma water appears to be the driving force for parotid and submaxillary secretion of drugs, but $p \mathrm{~K}_{\mathrm{a}}$ and molecular weight play roles in salivary secretion also (14a). The saliva/plasma concentration ratios vary from 0 to 1.0 (14a). The total volume of distribution of diffusible substances such as mannitol, inulin, and sucrose in man averages about $15-18 \%$ of body weight, or about $10.5-12.6$ liters for a $70-\mathrm{kg}$ man. One might expect the volumes of distribution of many free (unbound) drugs to be similar, and calculations made by the author for warfarin agree with this. Hence, even when the saliva/plasma concentration ratio is unity, a relatively small pool of drug is involved in the saliva cycle relative to the total amount of drug in the body. However, this cycle is one mechanism which cause drug to be returned from the circulation to the gastrointestinal tract. 
The enterohepatic cycle also contributes to return of drug from the circulation to the gastrointestinal tract. However, gallbladder emptying is a discontinuous process and to a large extent is stimulated by the eating of food. Thus one might not expect that drug involved in an enterohepatic cycle to be explicable on the basis of simple first-order kinetics as is assumed in the models of either Fig. 1 or Fig. 2.

\section{Models of Fig. 2}

Solutions for the models shown in Fig. 2 may readily be obtained by use of Laplace transforms. However, there is no separation of input and disposition functions, and the general equations of Benet (1) and of Vaughan and Trainor (2) cannot be used. Mathematically, each of these models requires that the polyexponential equation describing the plasma concentration curve following intravenous administration have the same number of exponential terms as the equation describing the plasma concentration curve following oral administration. It is the experience of this author, and many who have communicated with him, that in the evaluation of real data this observation is occasionally made and has not been explained to date. Such observations do not appear to have been reported in the literature since they do not agree with current theory, based on models such as shown in Fig. 1. Also, evaluation of data from panels of subjects or patients, given the same drug in the same dosage form by the same route of administration, often yields best-fit polyexponential equations with the number of terms varying from subject to subject. It is shown in this article that one and sometimes two of the polyexponential terms in the intravenous, but not the oral, equation essentially vanish and often cannot be "seen." Sometimes one of the terms in the oral equation also appears to vanish. A similar situation with classical linear pharmacokinetic equations is discussed by Ronfeld and Benet (37), but the phenomenon that they discuss applies to the oral equations and is different than that discussed in this article.

The implications of the models shown in Fig. 2 and the vanishing exponential terms are important and far-reaching. Some of these are as follows. (a) It may be very difficult to determine whether one is dealing with a one- or two-compartment open disposition model in the classical sense. (b) It may be very difficult to determine whether one is dealing with a two- or three-compartment open disposition model in the classical sense. (c) The largest or second-largest rate parameter in the polyexponential equation obtained after oral administration may not be an "absorption rate constant" at all. For all models shown in Fig. 2, the observable rate parameters are eigenvalues and are a function of all microscopic rate constants in the system. For these models, the actual absorption rate constants are obtained from the coefficients, not the exponents, of the polyexponential equations. 
(d) The Loo-Riegelman method (26) often may not yield "intrinsic absorption rate constants" at all, but rather an eigenvalue which is a root of a polynomial equation and a function of several rate constants.

Thus it appears to be more desirable to use so-called modelindependent pharmacokinetic equations rather than to solve for microscopic rate constants for some assumed specific model. One article (27) has emphasized some of these equations. The so-called model-independent methods are actually based on an " $n$ "-compartment mammillary model with central compartment elimination only. When one equates plasma clearance with total body clearance one is making the same assumption with respect to such a general mammillary model. By fitting data to simple polyexponential equations and using the numerical values of the coefficients and exponents, one can obtain essentially all the same desired parameters and solve essentially the same problems as those obtained by the more laborious procedure of deriving microscopic rate constants for some assumed model.

\section{THEORETICAL}

\section{Symbolism and Nomenclature}

$\lambda_{i}(i=1, \ldots, 4)$ are eigenvalues and are the roots of a polynomial equation and a function of all or most of the microscopic rate constants in the model. Arbitrarily $\lambda_{4}>\lambda_{3}>\lambda_{2}>\lambda_{1}$, hence $\lambda_{1}$ is the first rate parameter "peeled" from a set of $C_{p}, t$ data, $\lambda_{2}$ is the second one, etc.

$I_{i}(i=1, \ldots, 4)$ is a symbol for the coefficient of the polyexponential equation, replacing the more complicated forms which are functions of the $E_{i}$ 's, $\lambda_{i}$ 's, and $k_{i j}$ 's. $I_{i}^{\text {ivv. }}$ refers to bolus intravenous administration and $I_{i}^{\text {p.o. }}$ refers to oral administration.

$k_{i j}$ is a first-order rate constant for transfer of drug from compartment $i$ to compartment $j$.

$E_{i}(i=1, \ldots, 4)$ is used to represent the sum of all exit microscopic rate constants from the $i$ th compartment.

$D_{\text {i.v. }}$ is the dose administered by bolus intravenous administration.

$D_{\text {p.o. }}$ is the dose administered orally.

$F^{*}$ is the availability symbol used for the first-pass effect, such that $1-F^{*}$ is the portion of the available dose lost because of the firstpass effect following oral administration. For all models shown in Figs. 1 and $2, F^{*} \leq 1$, and $F^{*}$ is defined by equation 8 when $F=1$. 
$F$ is the availability symbol used to represent the fraction of the dose which reaches the circulation after oral administration either in the absence of the first-pass effect $\left(F^{*}=1\right)$ or after correction for the first-pass effect.

$V_{p}$ is the volume of the reference or "plasma compartment" where drug would be measured.

$\left(V_{p}\right)$ written below a compartment indicates which is the reference compartment.

$C_{0}=D_{\text {i.v. }} / V_{p}$.

$C_{0}^{\prime}=F D_{\text {p.o. }} / V_{p}$.

$C_{p}^{\text {i.v. }}$ is the concentration of unchanged drug in the reference (plasma) compartment at time $t$ following bolus intravenous injection.

$C_{p}^{\text {p.o. }}$ is the concentration of unchanged drug in the reference (plasma) compartment at time $t$ following oral administration.

$A_{t} / V_{p}$ is the cumulative amount of unchanged drug which has reached the reference (plasma) compartment in time $t$ divided by the volume $V_{p}$. This is the quantity calculated by application of the LooRiegelman method.

$\gamma, \delta$ are rate parameters appearing as exponents in the generalized equation obtained by resolving $A_{t} / V_{p}$ data.

\section{Models}

The six nonclassical models considered are shown in Fig. 2. Possible physiological interpretation of the various compartments is discussed in the Introduction.

\section{Equations}

For each of the six models shown in Fig. 2, solutions for $C_{p}^{\text {i.v. }}$ and $C_{p}^{\text {p.o. }}$ were obtained by use of Laplace transforms and the Heaviside expansion formula (1). For all of the $C_{p}^{\text {i.v. }}$ equations, feasible "collapsed equations" are also shown. The latter resulted from both the theoretical considerations and random number examples shown in the experimental sections.

Model I-A

$$
C_{p}^{\mathrm{i}, \mathrm{v} .}=C_{0} \frac{\left(k_{12}-\lambda_{1}\right)}{\left(\lambda_{2}-\lambda_{1}\right)} e^{-\lambda_{1} t}+C_{0} \frac{\left(k_{12}-\lambda_{2}\right)}{\left(\lambda_{1}-\lambda_{2}\right)} e^{-\lambda_{2} t}
$$

When $k_{12}$ is large relative to $k_{21}$ and/or $k_{20}$, then $\lambda_{2} \rightarrow k_{12}$ and the second term of equation 1 essentially vanishes. Not only does the second coefficient become very small but also $e^{-\lambda_{2} t}$ will be small from some time 
shortly after bolus injection of the dose. Hence the "collapsed equation" is shown in equation 2 .

$$
C_{p}^{\text {i.v. }}=C_{0} \frac{\left(k_{12}-\lambda_{1}\right)}{\left(\lambda_{2}-\lambda_{1}\right)} e^{-\lambda_{1} t}
$$

Also,

$$
C_{p}^{\text {p.o. }}=C_{0}^{\prime} \frac{\left(k_{12}\right)}{\left(\lambda_{2}-\lambda_{1}\right)}\left\{e^{-\lambda_{2} t}-e^{-\lambda_{2} t}\right\}
$$

In equations $1-3, \lambda_{1}$ and $\lambda_{2}$ are roots of a quadratic equation such that

$$
\begin{gathered}
\lambda_{1}+\lambda_{2}=k_{12}+k_{21}+k_{20} \\
\lambda_{1} \lambda_{2}=k_{12} k_{20}
\end{gathered}
$$

Equations 2 and 3 may be written as equations 6 and 7.

$$
\begin{aligned}
& C_{p}^{\text {i.v. }}=I_{1}^{\text {i.v. }} \cdot e^{-\lambda_{1} t} \\
& C_{p}^{\text {p.o. }}=I_{1}^{\text {p.o.o. }}\left\{e^{-\lambda_{1} t}-e^{-\lambda_{2} t}\right\}
\end{aligned}
$$

If one obtained numerical forms of equations 6 and 7 by appiication of the back-projection technique (15b) or by the computer-fitting of data, one would usually assume that the model for oral administration was the one-compartment open model with first-order absorption (see first model in Fig. 1) in which $\lambda_{1}=k_{20}, \lambda_{2}=k_{12}$, and $k_{21}=0$. However, the actual model may be model I-A.

Now, in general,

$$
F F^{*}=\frac{D_{\text {i.v. }} \cdot \int_{0}^{\infty} C_{p}^{\text {p.o. }} d t}{D_{\text {p.o. }} \cdot \int_{0}^{\infty} C_{p}^{\text {i.v. }} d t}
$$

But, for model I-A, $F^{*}=1{ }^{3}$ hence equation 8 yields the value of $F$, which is also equal to $C_{0}^{\prime} / C_{0}$.

Also,

$$
k_{12}=\frac{\left(\lambda_{2}-\lambda_{1}\right) I_{1}^{\text {p.o. }}}{C_{0}^{\prime}}
$$

Hence if data obey model I-A one may obtain $F$ and $k_{12}$ if both intravenous and oral data are available. It should also be noted, however, that the absorption rate constant, $k_{12}$, is obtained from the coefficient, $I_{1}^{\text {p.o. }}$, and that it is not equal to $\lambda_{2}$, which is the largest of the rate parameters

${ }^{3}$ It is interesting that when compartment 2 of model I-A is the reference compartment there is no first-pass effect, but when compartment 1 is the reference compartment there is a first-pass effect such that $F^{*}=k_{21} /\left(k_{21}+k_{20}\right)$. 
appearing as exponents in the polyexponential equation describing the plasma concentration after oral administration.

\section{Model I-B}

$$
\begin{aligned}
C_{p}^{\text {i.v. }}= & \frac{C_{0}\left[\left(k_{12}-\lambda_{1}\right)\left(E_{2}-\lambda_{1}\right)-k_{12} k_{21}\right]}{\left(\lambda_{2}-\lambda_{1}\right)\left(\lambda_{3}-\lambda_{1}\right)} e^{-\lambda_{1} t} \\
& +\frac{C_{0}\left[\left(k_{12}-\lambda_{2}\right)\left(E_{2}-\lambda_{2}\right)-k_{12} k_{21}\right]}{\left(\lambda_{1}-\lambda_{2}\right)\left(\lambda_{3}-\lambda_{2}\right)} e^{-\lambda_{2} t} \\
& +\frac{C_{0}\left[\left(k_{12}-\lambda_{3}\right)\left(E_{2}-\lambda_{3}\right)-k_{12} k_{21}\right]}{\left(\lambda_{1}-\lambda_{3}\right)\left(\lambda_{2}-\lambda_{3}\right)} e^{-\lambda_{3} t}
\end{aligned}
$$

Under certain conditions, the third term of equation 10 can vanish to yield the "collapsed equation" 11.

$$
\begin{aligned}
C_{p}^{\text {i.v. }}= & \frac{C_{0}\left[\left(k_{12}-\lambda_{1}\right)\left(E_{2}-\lambda_{1}\right)-k_{12} k_{21}\right]}{\left(\lambda_{2}-\lambda_{1}\right)\left(\lambda_{3}-\lambda_{1}\right)} e^{-\lambda_{1} t} \\
& +\frac{C_{0}\left[\left(k_{12}-\lambda_{2}\right)\left(E_{2}-\lambda_{2}\right)-k_{12} k_{21}\right]}{\left(\lambda_{1}-\lambda_{2}\right)\left(\lambda_{3}-\lambda_{2}\right)} e^{-\lambda_{2} t}
\end{aligned}
$$

Also,

$$
C_{p}^{\text {p.o. }}=\frac{k_{12} k_{23} C_{0}^{\prime} e^{-\lambda_{1} t}}{\left(\lambda_{2}-\lambda_{1}\right)\left(\lambda_{3}-\lambda_{1}\right)}+\frac{k_{12} k_{23} C_{0}^{\prime} e^{-\lambda_{2} t}}{\left(\lambda_{1}-\lambda_{2}\right)\left(\lambda_{3}-\lambda_{2}\right)}+\frac{k_{12} k_{23} C_{0}^{\prime} e^{-\lambda_{3} t}}{\left(\lambda_{1}-\lambda_{3}\right)\left(\lambda_{2}-\lambda_{3}\right)}
$$

Equations 10-12 may be written as equations 13 through 15 .

$$
\begin{aligned}
C_{p}^{\text {i.v. }}= & I_{1}^{\text {i.v. }} \cdot e^{-\lambda_{1} t}+I_{2}^{\text {i.v. }} \cdot e^{-\lambda_{2} t}+I_{3}^{\text {i.v. }} \cdot e^{-\lambda_{3} t} \\
& C_{p}^{\text {i.v. }}=I_{1}^{\text {i.v. }} \cdot e^{-\lambda_{1} t}+I_{2}^{\text {i.v. }} \cdot e^{-\lambda_{2} t} \\
C_{p}^{\text {p.o. }}= & I_{1}^{\text {p.o. }} \cdot e^{-\lambda_{1} t}+I_{2}^{\text {p.o. }} \cdot e^{-\lambda_{2} t}+I_{3}^{\text {p.o. }} \cdot e^{-\lambda_{3} t}
\end{aligned}
$$

If one obtained numerical equations corresponding to equations 14 and 15 , one would usually assign the two-compartment open model with firstorder absorption (i.e., the third model in Fig. 1), but the model may be model I-B.

Now, for model I-B

$$
\begin{aligned}
& F^{*}=1 \\
& F=C_{0}^{\prime} / C_{0}
\end{aligned}
$$

and equation 8 also applies.

It should also be noted that model I-B is one of the 21 "threecompartment open models with bolus intravenous injection" in the classical 
sense if the numbering of the compartments is altered such that compartment 3 becomes compartment 1 and vice versa.

In equations 10-15, $\lambda_{1}, \lambda_{2}$, and $\lambda_{3}$ are the absolute values of the roots of a cubic equation, and thus are complex functions of all the microscopic rate constants $k_{12}, k_{21}, k_{23}, k_{32}$, and $k_{30}$. If $k_{21}=0$, the situation would be the same except that the meanings of the rate parameters $\lambda_{1}, \lambda_{2}$, and $\lambda_{3}$ would be different.

By matching coefficients in equations 12 and 15 , one may derive equation 18 , which could provide a method of obtaining the absorption rate constant

$$
k_{23}=\frac{\left(k_{12}-\lambda_{1}\right)\left(\lambda_{2}-k_{12}\right)\left(I_{1}^{\text {p.o. }}+I_{2}^{\text {p.o. }}\right)}{k_{12} C_{0}^{\prime}}
$$

if both intravenous and oral data were available and model I-B applied.

\section{Model I-C}

$$
\begin{aligned}
C_{p}^{\text {i.v. }}= & \left\{\frac{C_{0}\left[\left(E_{1}-\lambda_{1}\right)\left(E_{2}-\lambda_{1}\right)-k_{12} k_{21}\right]}{\left(\lambda_{2}-\lambda_{1}\right)\left(\lambda_{3}-\lambda_{1}\right)}\right\} e^{-\lambda_{1} t} \\
& +\left\{\frac{C_{0}\left[\left(E_{1}-\lambda_{2}\right)\left(E_{2}-\lambda_{2}\right)-k_{12} k_{21}\right]}{\left(\lambda_{1}-\lambda_{2}\right)\left(\lambda_{3}-\lambda_{2}\right)}\right\} e^{-\lambda_{2} t} \\
& +\left\{\frac{C_{0}\left[\left(E_{1}-\lambda_{3}\right)\left(E_{2}-\lambda_{3}\right)-k_{12} k_{21}\right]}{\left(\lambda_{1}-\lambda_{3}\right)\left(\lambda_{2}-\lambda_{3}\right)}\right\} e^{-\lambda_{2} t}
\end{aligned}
$$

where $E_{1}=k_{12}+k_{13}, E_{2}=k_{21}+k_{23}$, and $\lambda_{1}, \lambda_{2}$, and $\lambda_{3}$ are roots of a cubic equation and a function of all the $k_{i j}$ 's.

For the same reasons as given for model I-B, feasible "collapsed equations" are equations 20 and 21 .

$$
\begin{aligned}
C_{p}^{\text {i.v. }}= & \left\{\frac{C_{0}\left[\left(E_{1}-\lambda_{1}\right)\left(E_{2}-\lambda_{1}\right)-k_{12} k_{21}\right]}{\left(\lambda_{2}-\lambda_{1}\right)\left(\lambda_{3}-\lambda_{1}\right)}\right\} e^{-\lambda_{1} t} \\
& +\left\{\frac{C_{0}\left[\left(E_{1}-\lambda_{2}\right)\left(E_{2}-\lambda_{2}\right)-k_{12} k_{21}\right]}{\left(\lambda_{1}-\lambda_{2}\right)\left(\lambda_{3}-\lambda_{2}\right)}\right\} e^{-\lambda_{2} t} \\
C_{p}^{\text {i.v. }}= & \left\{\frac{C_{0}\left[\left(E_{1}-\lambda_{1}\right)\left(E_{2}-\lambda_{1}\right)-k_{12} k_{21}\right]}{\left(\lambda_{2}-\lambda_{1}\right)\left(\lambda_{3}-\lambda_{1}\right)}\right\} e^{-\lambda_{1} t}
\end{aligned}
$$

Also,

$$
\begin{aligned}
C_{p}^{\text {p.o. }}= & C_{0}^{\prime}\left\{\frac{k_{12} k_{23}+k_{13}\left(E_{2}-\lambda_{1}\right)}{\left(\lambda_{2}-\lambda_{1}\right)\left(\lambda_{3}-\lambda_{1}\right)}\right\} e^{-\lambda_{1} t}+C_{0}^{\prime}\left\{\frac{k_{12} k_{23}+k_{13}\left(E_{2}-\lambda_{2}\right)}{\left(\lambda_{1}-\lambda_{2}\right)\left(\lambda_{3}-\lambda_{2}\right)}\right\} e^{-\lambda_{2} t} \\
& +C_{0}\left\{\frac{k_{12} k_{23}+k_{13}\left(E_{2}-\lambda_{3}\right)}{\left(\lambda_{1}-\lambda_{3}\right)\left(\lambda_{2}-\lambda_{3}\right)}\right\} e^{-\lambda_{3} t}
\end{aligned}
$$


Similar equations as equations 13-14 may be written in place of equations 20 and 21 and the same arguments presented as given below equation 15 . Thus we could readily assign the wrong model.

It should be noted that model I-C is also one of the 21 classical "three-compartment open models with bolus intravenous injection" if the numbering of compartments is altered such that 3 becomes 1 and 1 becomes 3 .

Now for model I-C equation 8 applies and $F^{*}$ is given by equation 23 .

$$
F^{*}=\frac{k_{13} E_{2}+k_{12} k_{23}}{E_{1} E_{3}-k_{12} k_{21}}
$$

Model II-A

$$
\begin{aligned}
C_{p}^{\text {i.v. }}= & C_{0}\left\{\frac{\left(k_{12}-\lambda_{1}\right)\left(E_{3}-\lambda_{1}\right)}{\left(\lambda_{2}-\lambda_{1}\right)\left(\lambda_{3}-\lambda_{1}\right)}\right\} e^{-\lambda_{1} t}+C_{0}\left\{\frac{\left(k_{12}-\lambda_{2}\right)\left(E_{3}-\lambda_{2}\right)}{\left(\lambda_{1}-\lambda_{2}\right)\left(\lambda_{3}-\lambda_{2}\right)}\right\} e^{-\lambda_{2} t} \\
& +C_{0}\left\{\frac{\left(k_{12}-\lambda_{3}\right)\left(E_{3}-\lambda_{3}\right)}{\left(\lambda_{1}-\lambda_{3}\right)\left(\lambda_{2}-\lambda_{3}\right)}\right\} e^{-\lambda_{3} t}
\end{aligned}
$$

where $E_{3}=k_{32}+k_{30}$ and $\lambda_{1}, \lambda_{2}$, and $\lambda_{3}$ are the roots of a cubic equation and a function of all the $k_{i j}$ 's.

When $k_{12}$ is large relative to the other microscopic rate constants, then $\lambda_{3} \rightarrow k_{12}$ and $e^{-\lambda_{3} t}$ is small even for times shortly after administration, hence the third term of equation 24 tends to vanish and the "collapsed equation" is equation 25 .

$$
C_{p}^{\text {i.v. }}=C_{0}\left\{\frac{\left(k_{12}-\lambda_{1}\right)\left(E_{3}-\lambda_{1}\right)}{\left(\lambda_{2}-\lambda_{1}\right)\left(\lambda_{3}-\lambda_{1}\right)}\right\} e^{-\lambda_{1} t}+C_{0}\left\{\frac{\left(k_{12}-\lambda_{2}\right)\left(E_{3}-\lambda_{2}\right)}{\left(\lambda_{1}-\lambda_{2}\right)\left(\lambda_{3}-\lambda_{2}\right)}\right\} e^{-\lambda_{3} t}
$$

Under certain conditions, the second and third terms of equation 24 could vanish and the second feasible "collapsed equation" is equation 26.

$$
C_{p}^{\text {i.v. }}=C_{0}\left\{\frac{\left(k_{12}-\lambda_{1}\right)\left(E_{3}-\lambda_{1}\right)}{\left(\lambda_{2}-\lambda_{1}\right)\left(\lambda_{3}-\lambda_{1}\right)}\right\} e^{-\lambda_{2} t}
$$

Also,

$$
\begin{aligned}
C_{p}^{\text {p.o. }}= & C_{0}^{\prime}\left\{\frac{k_{12}\left(E_{3}-\lambda_{1}\right)}{\left(\lambda_{2}-\lambda_{1}\right)\left(\lambda_{3}-\lambda_{1}\right)}\right\} e^{-\lambda_{1} t}+C_{0}^{\prime}\left\{\frac{k_{12}\left(E_{3}-\lambda_{2}\right)}{\left(\lambda_{1}-\lambda_{2}\right)\left(\lambda_{3}-\lambda_{2}\right)}\right\} e^{-\lambda_{2} t} \\
& +C_{0}^{\prime}\left\{\frac{k_{12}\left(E_{3}-\lambda_{3}\right)}{\left(\lambda_{1}-\lambda_{3}\right)\left(\lambda_{2}-\lambda_{3}\right)}\right\} e^{-\lambda_{3} t}
\end{aligned}
$$

Equations 24-27 may be written as equations 28-31 where the meanings of the coefficients may be seen by matching. 


$$
\begin{aligned}
& C_{p}^{\text {i.v. }}=I_{1}^{\mathrm{i} . v \mathrm{v}} \cdot e^{-\lambda_{1} t}+I_{2}^{\mathrm{i} . \mathrm{v}} \cdot e^{-\lambda_{2} t}+I_{3}^{\mathrm{i} . \mathrm{v} .} \cdot e^{-\lambda_{3} t} \\
& C_{p}^{\text {i.v. }}=I_{1}^{\mathrm{i} . \mathrm{v} .} \cdot e^{-\lambda_{1} t}+I_{2}^{\mathrm{i} . \mathrm{v} .} \cdot e^{-\lambda_{2} t} \\
& C_{p}^{\text {i.v. }}=I_{1}^{\mathrm{i} . \mathrm{v} .} \cdot e^{-\lambda_{1} t} \\
& C_{p}^{\text {p.o. }}=I_{1}^{\text {p.o. }} \cdot e^{-\lambda_{1} t}+I_{2}^{\text {p.o. }} \cdot e^{-\lambda_{2} t}+I_{3}^{\text {p.o. }} \cdot e^{-\lambda_{3} t}
\end{aligned}
$$

One may also readily obtain equations 32 and 33

$$
\begin{aligned}
& k_{12}=\frac{\lambda_{1}}{1-\left(F \cdot I_{1}^{\text {i.v. }}\right) / I_{1}^{\text {p.o. }}} \\
& k_{12}=\frac{\lambda_{2}}{1-\left(F \cdot I_{2}^{\text {i.v. }}\right) / I_{2}^{\text {p.o. }}}
\end{aligned}
$$

which could provide a method of obtaining the absorption rate constant, $k_{12}$, since $F$ is given by equation 8 and for this model $F^{*}=1$ when the reference compartment is compartment 2 .

If data were fitted to equations 29 and 31, the classical assignment would be a special case of model II-A in which $k_{21}=0$ (i.e., the third model in Fig. 1). However, the actual model may be model II-A in which $k_{21} \neq 0$.

\section{Model II-B}

$$
\begin{aligned}
C_{p}^{\text {i.v. }}= & C_{0}\left\{\frac{\left(E_{4}-\lambda_{1}\right)\left[\left(k_{12}-\lambda_{1}\right)\left(E_{2}-\lambda_{1}\right)-k_{12} k_{21}\right]}{\left(\lambda_{2}-\lambda_{1}\right)\left(\lambda_{3}-\lambda_{1}\right)\left(\lambda_{4}-\lambda_{1}\right)}\right\} e^{-\lambda_{1} t} \\
& +C_{0}\left\{\frac{\left(E_{4}-\lambda_{2}\right)\left[\left(k_{12}-\lambda_{2}\right)\left(E_{2}-\lambda_{2}\right)-k_{12} k_{21}\right]}{\left(\lambda_{1}-\lambda_{2}\right)\left(\lambda_{3}-\lambda_{2}\right)\left(\lambda_{4}-\lambda_{2}\right)}\right\} e^{-\lambda_{2} t} \\
& +C_{0}\left\{\frac{\left(E_{4}-\lambda_{2}\right)\left[\left(k_{12}-\lambda_{3}\right)\left(E_{2}-\lambda_{3}\right)-k_{12} k_{21}\right]}{\left(\lambda_{1}-\lambda_{3}\right)\left(\lambda_{2}-\lambda_{3}\right)\left(\lambda_{4}-\lambda_{3}\right)}\right\} e^{-\lambda_{3} t} \\
& +C_{0}\left\{\frac{\left(E_{4}-\lambda_{4}\right)\left[\left(k_{12}-\lambda_{4}\right)\left(E_{2}-\lambda_{4}\right)-k_{12} k_{21}\right]}{\left(\lambda_{1}-\lambda_{4}\right)\left(\lambda_{2}-\lambda_{4}\right)\left(\lambda_{3}-\lambda_{4}\right)}\right\} e^{-\lambda_{4} t}
\end{aligned}
$$

where $E_{4}=k_{43}+k_{40}, E_{2}=k_{21}+k_{23}$, and the $\lambda_{i}$ 's are the roots of a fourthdegree polynomial and a function of all the $k_{i j}$ 's. 
Depending on the parameter values, either or both of the $e^{-\lambda_{3} t}$ and $e^{-\lambda_{4} t}$ terms may vanish, hence feasible "collapsed equations" are equations 35-37.

$$
\begin{aligned}
C_{p}^{\text {i.v. }}= & C_{0}\left\{\frac{\left(E_{4}-\lambda_{1}\right)\left[\left(k_{12}-\lambda_{1}\right)\left(E_{2}-\lambda_{1}\right)-k_{12} k_{21}\right]}{\left(\lambda_{2}-\lambda_{1}\right)\left(\lambda_{3}-\lambda_{1}\right)\left(\lambda_{4}-\lambda_{1}\right)}\right\} e^{-\lambda_{1} t} \\
& +C_{0}\left\{\frac{\left(E_{4}-\lambda_{2}\right)\left[\left(k_{12}-\lambda_{2}\right)\left(E_{2}-\lambda_{2}\right)-k_{12} k_{21}\right]}{\left(\lambda_{1}-\lambda_{2}\right)\left(\lambda_{3}-\lambda_{2}\right)\left(\lambda_{4}-\lambda_{2}\right)}\right\} e^{-\lambda_{2} t} \\
& +C_{0}\left\{\frac{\left(E_{4}-\lambda_{3}\right)\left[\left(k_{12}-\lambda_{3}\right)\left(E_{2}-\lambda_{3}\right)-k_{12} k_{21}\right]}{\left(\lambda_{1}-\lambda_{3}\right)\left(\lambda_{2}-\lambda_{3}\right)\left(\lambda_{4}-\lambda_{3}\right)}\right\} e^{-\lambda_{3} t} \\
C_{p}^{\text {i.v. }}= & C_{0}\left\{\frac{\left(E_{4}-\lambda_{1}\right)\left[\left(k_{12}-\lambda_{1}\right)\left(E_{2}-\lambda_{1}\right)-k_{12} k_{21}\right]}{\left(\lambda_{2}-\lambda_{1}\right)\left(\lambda_{3}-\lambda_{1}\right)\left(\lambda_{4}-\lambda_{1}\right)}\right\} e^{-\lambda_{1} t} \\
& +C_{0}\left\{\frac{\left(E_{4}-\lambda_{2}\right)\left[\left(k_{12}-\lambda_{2}\right)\left(E_{2}-\lambda_{2}\right)-k_{12} k_{21}\right]}{\left(\lambda_{1}-\lambda_{2}\right)\left(\lambda_{3}-\lambda_{2}\right)\left(\lambda_{4}-\lambda_{2}\right)}\right\} e^{-\lambda_{2} t} \\
& +C_{0}\left\{\frac{\left(E_{4}-\lambda_{4}\right)\left[\left(k_{12}-\lambda_{4}\right)\left(E_{2}-\lambda_{4}\right)-k_{12} k_{21}\right]}{\left(\lambda_{1}-\lambda_{4}\right)\left(\lambda_{2}-\lambda_{4}\right)\left(\lambda_{3}-\lambda_{4}\right)}\right\} e^{-\lambda_{4} t} \\
C_{p}^{\text {i.v. }}= & C_{0}\left\{\frac{\left(E_{4}-\lambda_{1}\right)\left[\left(k_{12}-\lambda_{1}\right)\left(E_{2}-\lambda_{1}\right)-k_{12} k_{21}\right]}{\left(\lambda_{2}-\lambda_{1}\right)\left(\lambda_{3}-\lambda_{1}\right)\left(\lambda_{4}-\lambda_{1}\right)}\right\} e^{-\lambda_{1} t} \\
& +C_{0}\left\{\frac{\left(E_{4}-\lambda_{2}\right)\left[\left(k_{12}-\lambda_{2}\right)\left(E_{2}-\lambda_{2}\right)-k_{12} k_{21}\right]}{\left(\lambda_{1}-\lambda_{2}\right)\left(\lambda_{3}-\lambda_{2}\right)\left(\lambda_{4}-\lambda_{2}\right)}\right\} e^{-\lambda_{2} t}
\end{aligned}
$$

Also,

$$
\begin{aligned}
C_{p}^{\text {p.o. }}= & C_{0}^{\prime}\left\{\frac{k_{12} k_{23}\left(E_{4}-\lambda_{1}\right)}{\left(\lambda_{2}-\lambda_{1}\right)\left(\lambda_{3}-\lambda_{1}\right)\left(\lambda_{4}-\lambda_{1}\right)}\right\} e^{-\lambda_{1} t} \\
& +C_{0}^{\prime}\left\{\frac{k_{12} k_{23}\left(E_{4}-\lambda_{2}\right)}{\left(\lambda_{1}-\lambda_{2}\right)\left(\lambda_{3}-\lambda_{2}\right)\left(\lambda_{4}-\lambda_{2}\right)}\right\} e^{-\lambda_{2} t} \\
& +C_{0}^{\prime}\left\{\frac{k_{12} k_{23}\left(E_{4}-\lambda_{3}\right)}{\left(\lambda_{1}-\lambda_{3}\right)\left(\lambda_{2}-\lambda_{3}\right)\left(\lambda_{4}-\lambda_{3}\right)}\right\} e^{-\lambda_{3} t} \\
& +C_{0}^{\prime}\left\{\frac{k_{12} k_{23}\left(E_{4}-\lambda_{4}\right)}{\left(\lambda_{1}-\lambda_{4}\right)\left(\lambda_{2}-\lambda_{4}\right)\left(\lambda_{3}-\lambda_{4}\right)}\right\} e^{-\lambda_{4} t}
\end{aligned}
$$

Equation 38 may be written as equation 39 .

$$
C_{p}^{\text {p.o. }}=I_{1}^{\text {p.o. }} \cdot e^{-\lambda_{1} t}+I_{2}^{\text {p.o. }} \cdot e^{-\lambda_{2} t}+I_{3}^{\text {p.o. }} \cdot e^{-\lambda_{3} t}+I_{4}^{\text {p.o. }} \cdot e^{-\lambda_{4} t}
$$


If oral data are "stripped" or fitted by nonlinear least squares to provide numerical values for equation 39 , then a preliminary estimate of $E_{4}$ may be obtained with equation 40 .

$$
E_{4}=\left(\lambda_{1}+R \lambda_{2}\right) /(1+R)
$$

where

$$
R=\frac{\left(\lambda_{3}-\lambda_{1}\right)\left(\lambda_{4}-\lambda_{1}\right) I_{1}^{\text {p.o. }}}{\left(\lambda_{3}-\lambda_{2}\right)\left(\lambda_{4}-\lambda_{2}\right) I_{2}^{\text {p.o. }}}
$$

An estimate of $k_{12} k_{23} C_{0}^{\prime}$ could then be obtained with equation 42 .

$$
k_{12} k_{23} C_{0}^{\prime}=\frac{\left(\lambda_{2}-\lambda_{1}\right)\left(\lambda_{3}-\lambda_{1}\right)\left(\lambda_{4}-\lambda_{1}\right) I_{1}^{\text {p.o. }}}{\left(E_{4}-\lambda_{1}\right)}
$$

Oral data could then be fitted to equation 38 and $k_{12} k_{23} C_{0}^{\prime}, E_{4}, \lambda_{1}, \lambda_{2}, \lambda_{3}$, and $\lambda_{4}$ could then be the parameters estimated.

Model II-C

$$
\begin{aligned}
C_{p}^{\text {i.v. }}= & C_{0}\left\{\frac{\left(E_{4}-\lambda_{1}\right)\left[\left(E_{1}-\lambda_{1}\right)\left(E_{2}-\lambda_{1}\right)-k_{12} k_{21}\right]}{\left(\lambda_{2}-\lambda_{1}\right)\left(\lambda_{3}-\lambda_{1}\right)\left(\lambda_{4}-\lambda_{1}\right)}\right\} e^{-\lambda_{1} t} \\
& +C_{0}\left\{\frac{\left(E_{4}-\lambda_{2}\right)\left[\left(E_{1}-\lambda_{2}\right)\left(E_{2}-\lambda_{1}\right) k_{12} k_{21}\right]}{\left(\lambda_{1}-\lambda_{2}\right)\left(\lambda_{3}-\lambda_{2}\right)\left(\lambda_{4}-\lambda_{2}\right)}\right\} e^{-\lambda_{2} t} \\
& +C_{0}\left\{\frac{\left(E_{4}-\lambda_{3}\right)\left[\left(E_{1}-\lambda_{3}\right)\left(E_{2}-\lambda_{3}\right)-k_{12} k_{21}\right]}{\left(\lambda_{1}-\lambda_{3}\right)\left(\lambda_{2}-\lambda_{3}\right)\left(\lambda_{4}-\lambda_{3}\right)}\right\} e^{-\lambda_{3} t} \\
& +C_{0}\left\{\frac{\left(E_{4}-\lambda_{4}\right)\left[\left(E_{1}-\lambda_{4}\right)\left(E_{2}-\lambda_{4}\right)-k_{12} k_{21}\right]}{\left(\lambda_{1}-\lambda_{4}\right)\left(\lambda_{2}-\lambda_{4}\right)\left(\lambda_{3}-\lambda_{4}\right)}\right\} e^{-\lambda_{4} t}
\end{aligned}
$$

Equation 43 is the same as equation 34 except that $E_{1}$ replaces $k_{12}$. The significance is the same since for model II-B $E_{1}=k_{12}$ whereas for model II-C $E_{1}=k_{12}+k_{13}$. Hence the "collapsed equations" corresponding to equation 43 are the same as equations 35-37 except that $E_{1}$ replaces $k_{12}$. In equation $43, E_{4}=k_{43}+k_{40}$ and $E_{2}=k_{21}+k_{23}$ as in equation 34 .

$$
\begin{aligned}
C_{p}^{\text {p.o. }}= & C_{0}^{\prime}\left\{\frac{\left(E_{4}-\lambda_{1}\right)\left[k_{13}\left(E_{2}-\lambda_{1}\right)+k_{12} k_{23}\right]}{\left(\lambda_{2}-\lambda_{1}\right)\left(\lambda_{3}-\lambda_{1}\right)\left(\lambda_{4}-\lambda_{1}\right)}\right\} e^{-\lambda_{1} t} \\
& +C_{0}^{\prime}\left\{\frac{\left(E_{4}-\lambda_{2}\right)\left[k_{13}\left(E_{2}-\lambda_{2}\right)+k_{12} k_{23}\right]}{\left(\lambda_{1}-\lambda_{2}\right)\left(\lambda_{3}-\lambda_{2}\right)\left(\lambda_{4}-\lambda_{2}\right)}\right\} e^{-\lambda_{2} t} \\
& +C_{0}^{\prime}\left\{\frac{\left(E_{4}-\lambda_{3}\right)\left[k_{13}\left(E_{2}-\lambda_{3}\right)+k_{12} k_{23}\right]}{\left(\lambda_{1}-\lambda_{3}\right)\left(\lambda_{2}-\lambda_{3}\right)\left(\lambda_{4}-\lambda_{3}\right)}\right\} e^{-\lambda_{3} t} \\
& +C_{0}^{\prime}\left\{\frac{\left(E_{4}-\lambda_{4}\right)\left[k_{13}\left(E_{2}-\lambda_{4}\right)+k_{12} k_{23}\right]}{\left(\lambda_{1}-\lambda_{4}\right)\left(\lambda_{2}-\lambda_{4}\right)\left(\lambda_{3}-\lambda_{4}\right)}\right\} e^{-\lambda_{4} t}
\end{aligned}
$$




\section{Other Models}

Model I-B Type. When $k_{21}=0$ in model I-B, equations $10-17$ still apply.

Model I-C Type. Equations 19-22 also apply for varieties of model I-C in which (a) $k_{21}=0$, (b) $k_{32}=0$, (c) $k_{31}=0$, (d) $k_{21}=0$ and $k_{31}=0$, (e) $k_{21}=0$ and $k_{32}=0$, (f) $k_{31}=0$ and $k_{32}=0$, and (g) $k_{21}=0, k_{31}=0$, and $k_{32}=0$.

Model II-A Type. Equations 24-33 apply for varieties of model II-A in which (a) $k_{30}=0$ and (b) $k_{20}=0$.

Model II-B Type. Equations 34-42 apply for varieties of model II-B in which (a) $k_{21}=0$, (b) $k_{40}=0$, (c) $k_{30}=0$, (d) $k_{21}=0$ and $k_{40}=0$, and $k_{21}=0$ and $k_{30}=0$.

Model II-CType. Equations 43 and 44 apply for varieties of model II-C in which (a) $k_{21}=0$; (b) $k_{31}=0$; (c) $k_{32}=0$; (d) $k_{40}=0$; (e) $k_{30}=0$; (f) $k_{21}=0$ and $k_{40}=0$; (g) $k_{21}=0$ and $k_{30}=0$; (h) $k_{31}=0$ and $k_{40}=0$; (i) $k_{31}=0$ and $k_{30}=0$; (j) $k_{32}=0$ and $k_{40}=0$; (k) $k_{32}=0$ and $k_{30}=0$; (l) $k_{21}=0$ and $k_{30}=0 ;(\mathrm{m}) k_{21}=0$ and $k_{32}=0$; (n) $k_{21}=0, k_{31}=0$, and $k_{30}=0$; (o) $k_{21}=0$, $k_{32}=0$, and $k_{30}=0 ;(\mathrm{p}) k_{21}=0, k_{31}=0$, and $k_{40}=0 ;(\mathrm{q}) k_{21}=0, k_{32}=0$, and $k_{40}=0$; (r) $k_{31}=0, k_{32}=0$, and $k_{40}=0 ;$ (s) $k_{21}=0, k_{32}=0$, and $k_{40}=0$; (t) $k_{21}=0, k_{31}=0, k_{32}=0$, and $k_{40}=0$; and (u) $k_{21}=0, k_{31}=0, k_{32}=0$, and $k_{30}=0$.

\section{Application of the Wagner-Nelson and Loo-Riegelman Methods}

The Wagner-Nelson method (28) was applied to the equations for models I-A, I-B, and I-C. This involved application of equation 45.

$$
A_{t} / V_{p}=C_{p}^{\text {p.o. }}+\lambda_{1} \int_{0}^{t} C_{p}^{\text {p.o. }} \cdot d t^{\prime}
$$

The right-hand sides of equations 3,12 , and 22 were substituted for $C_{p}^{\text {p.o. }}$ in equation 45 for models I-A, I-B, and I-C, respectively. After simplifications, the results shown in Table II were obtained. The results show that if the $A_{t} / V_{p}, t$ data were "stripped" the observed rate parameters would not be absorption rate constants but $\lambda_{i}$ values. The absorption rate constants are, however, contained in the coefficients of the $e^{-\lambda_{1} t}$ or $e^{-\lambda_{2} t}$ term.

The Loo-Riegelman method (26) was applied to model II-A. The Loo-Riegelman method effectively measures the quantity shown in equation 46 for this model.

$$
A_{t} / V_{p}=C_{p}^{\text {p.o. }}+C_{3}^{\text {p.o. }}+k_{20} \int_{0}^{t} C_{p}^{\text {p.o. }} d t^{\prime}
$$




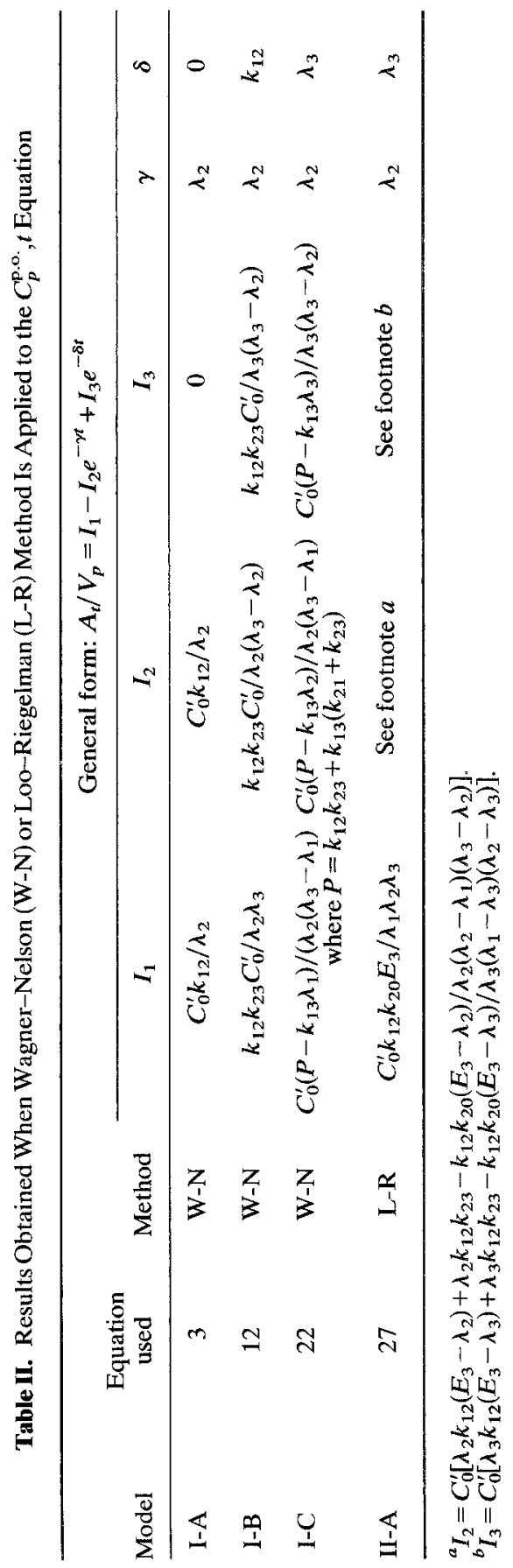


where $C_{3}^{\text {p.o. }}=A_{3}^{\text {p.o. }} / V_{p}$ and $A_{3}^{\text {p.o. }}$ is the amount of drug in compartment 3 at time $t$ after oral administration and the assumption is made that $k_{30}=0$. The right-hand side of equation 27 was substituted for $C_{p}^{\text {p.o. }}$, and the right-hand side of equation 47 was substituted for $C_{3}^{\text {p.o. }}$ in equation 46 .

$$
C_{3}^{\text {p.o. }}=\frac{k_{12} k_{23} C_{0}^{\prime} e^{-\lambda_{1} t}}{\left(\lambda_{2}-\lambda_{1}\right)\left(\lambda_{3}-\lambda_{1}\right)}+\frac{k_{12} k_{23} C_{0}^{\prime} e^{-\lambda_{2} t}}{\left(\lambda_{1}-\lambda_{2}\right)\left(\lambda_{3}-\lambda_{2}\right)}+\frac{k_{12} k_{23} C_{0}^{\prime} e^{-\lambda_{3} t}}{\left(\lambda_{1}-\lambda_{3}\right)\left(\lambda_{2}-\lambda_{3}\right)}
$$

After simplification, the result shown in Table II was obtained. Again the observed rate parameters are $\lambda_{i}$ values and not "intrinsic absorption rate constants." The absorption rate constant, $k_{12}$, for this model is contained in the coefficients of the equation for $A_{t} / V_{p}$ as a function of time.

The algebra was just too horrendous and no attempt was made to apply a similar equation as equation 46 to models II-B and II-C. However, intuitively, one can see that similar results would be obtained as those obtained with model II-A. The observed rate parameters would be $\lambda_{i}$ values and not "intrinsic absorption rate constants."

\section{EXPERIMENTAL}

Some examples are given to show that the $C_{p}^{\text {i.v. }}$ equations collapse readily and that sometimes collapsing also occurs in the $C_{p}^{\text {p.o. }}$ equations.

\section{Model II-A (Example 1)}

Values of $\lambda_{3}=4, k_{12}=3.75, \lambda_{2}=2, E_{3}=0.5, \lambda_{1}=0.1$, and $C_{0}=C_{0}^{\prime}=$ 100 were substituted into equations 24 and 27 to yield equations 48 and 49 , respectively.

$$
\begin{aligned}
& C_{p}^{\mathrm{i} . v .}=19.703 e^{-0.1 t}+69.079 e^{-2 t}+11.218 e^{-4 t} \\
& C_{p}^{\text {p.o. }}=20.243 e^{-0.1 t}+148.026 e^{-2 t}-168.269^{-4 t}
\end{aligned}
$$

Integration of equations 48 and 49 between the limits of $t=0$ and $t=\infty$ gave an area of 234.4 . The third term of equation 48 contributes only about $1 \%$ to the total area, while the third term of equation 49 makes a contribution of about $18 \%$.

Values of $t=0.125,0.25,0.5,0.75,1,2,4,6,8,12$, and $24 \mathrm{hr}$ were substituted into equations 48 and 49 to yield a set of $C_{p}^{\text {i.v. }}$ and a set of $C_{p}^{\text {p.o. }}$ values. The two sets were fitted with program CSTRIP (38) and a high-speed digital computer, and the results are given as equations 50 and 51 .

$$
\begin{aligned}
& C_{p}^{\hat{i . v .}}=20.292 e^{-0.1018 t}+78.174 e^{-2.1600 t} \\
& C_{p}^{\hat{\text { p.o. }}}=20.467 e^{-0.1002 t}+143.59 e^{-1.995 t}-163.83 e^{-4.129 t}
\end{aligned}
$$


The $r^{2}$ value

$$
r^{2}=\left[\sum_{i=1}^{n} C_{i}^{2}-\left(\sum_{i=1}^{n} C_{i}\right)^{2} / n-\sum_{i=1}^{n}\left(C_{i}-\hat{C}_{i}\right)^{2}\right] /\left[\sum_{i=1}^{n} C_{i}^{2}-\left(\sum_{i=1}^{n} C_{i}\right)^{2} / n\right]
$$

for the fit shown as equation 50 was 0.99995 and that for equation 51 was 0.99939 . The area estimated from equation 50 was $235.52(100.5 \%$ of theoretical) and that from equation 51 was 236.56 (100.9\% of theoretical). Similar results were achieved with a desk calculator using the backprojection technique (15b). Observed and estimated values are compared in the Appendix. This simulation clearly shows the phenomenon of the vanishing exponential term in the intravenous, but not the oral, equation. It should also be noted that at 1,2 , and 3 min the third term of equation 48 contributes only $0.21,0.004$, and $0.0001 \%$ of the $C_{p}^{\text {i.v. }}$ values, respectively.

\section{Model II-A (Example 2)}

Example 2 illustrates the effect of intestinal secretion on the pharmacokinetics of a non-plasma-protein-bound and non-tissue-bound drug and may indicate the possible magnitude of such effects on free (unbound) species in the presence of plasma protein and tissue binding. Volumes and rate constants have been chosen that are reasonably "physiological" for the dog. Compartment 1 of model II-A was assumed to have a volume of $225 \mathrm{ml}$ and was estimated using the value of $1500 \mathrm{ml}$ for upper intestinal contents in man $(22 \mathrm{a})$ and this was then reduced according to the ratio of body weights of the dog to man. The volume of compartment 2 was taken as $450 \mathrm{ml}$ (plasma volume of $4.5 \%$ of body weight for a $10-\mathrm{kg} \mathrm{dog}$ ). The volume of compartment 3 was taken as $1450 \mathrm{ml}$, which is the extracellular fluid volume of the dog. The glomerular filtration rate of the $\operatorname{dog}$ is $4.3 \mathrm{ml} / \mathrm{min} / \mathrm{kg}$, or $43 \mathrm{ml} / \mathrm{min}$ for a $10-\mathrm{kg} \mathrm{dog}$; with a plasma volume of $450 \mathrm{ml}$, this gives an elimination rate constant, $k_{20}$, equal to $5.7 \mathrm{hr}^{-1}$. Values of $k_{12}=6 \mathrm{hr}^{-1}$ and $k_{23}=2.4 \mathrm{hr}^{-1}$ were assumed. Based on Fick's law of diffusion, $k_{32}=$ $(450 / 1450) \times 2.4=0.745 \mathrm{hr}^{-1}$ and $k_{21}=(225 / 450) \times 6=3 \mathrm{hr}^{-1}$.

The above values and the appropriate Laplace transform equations led to equations 52 and 53 for $C_{\mathrm{p}}^{\text {i.v. }}$ and $C_{p}^{\text {p.o. }}$, respectively.

$$
\begin{aligned}
& C_{p}^{\text {i.v. }}=5.6950 e^{-0.5009 t}+38.1456 e^{-3.7384 t}+136.1097 e^{-13.6057 t}(52) \\
& C_{p}^{\text {p.o. }}=6.2137 e^{-0.5009 t}+101.200 e^{-3.7384 t}-107.4137 e^{-13.6057 t}(53)
\end{aligned}
$$

Integration of equation 52 between the limits of 0 and $\infty$ indicates that the first, second, and third terms of equation 52 contribute $36.0,32.3$, and $31.7 \%$ toward the total area of 31.57 , respectively. 
If one obtains the $C_{p}^{\text {d.v. }}$ equation corresponding to the same model except that $k_{21}=0$, then compartment 1 is not involved and the result is shown as equation 54 .

$$
C_{p}^{\mathrm{i} . v .}=5.4187 e^{-0.5094 t}+174.5813 e^{-8.3356 t}
$$

It should be noted that although the smallest of the eigenvalues in equations 52 and 54 are very similar, the eigenvalue of 8.3356 in equation 54 is intermediate between the two largest eigenvalues, namely 3.7384 and 13.6057 in equation 52. This does illustrate the possible importance of intestinal secretion.

\section{Model II-C}

Values of $\lambda_{4}=9, k_{23}=8.5, \lambda_{3}=8, E_{1}=6, k_{12}=5.5, \lambda_{2}=3, E_{4}=2$, $k_{13}=0.5, \lambda_{1}=0.25, k_{21}=0$, and $C_{0}=C_{0}^{\prime}=100$ were substituted into equations 43 and 44 to yield equations 55 and 56 , respectively.

$$
\begin{aligned}
& C_{p}^{\text {i.v. }}=44.516 e^{-0.25 t}+20.000 e^{-3 t}+15.484 e^{-8 t}+20.000 e^{-9 t} \\
& C_{p}^{\text {p.o. }}=47.742 e^{-0.25 t}+60.000 e^{-3 t}-727.742 e^{-8 t}+620.000 e^{-9 t}
\end{aligned}
$$

Integration of both equations 55 and 56 between the limits of $t=0$ and $t=\infty$ gave the same area of 188.89 (i.e., $F^{*}=1$ for model II-C). The third and fourth terms of equation 55 contribute only 1.0 and $1.2 \%$ respectively, toward this total area.

Values of $t=0.125,0.25,0.5,0.75,1,1.5,2,3,4,6,8,12$, and 24 hours were substituted into equations 55 and 56 to yield a set of $C_{p}^{\text {i.v. }}$ and a set of $C_{p}^{\text {p.o. }}$ values. The $C_{p}^{\text {i.v. }}, t$ set was fitted with the program CSTRIP and the digital computer. The $C_{p}^{\text {i.v. }}, t$ set was fitted to a four-term polyexponential equation with the program NONLIN (29) and a digital computer. Results are shown as equations 57 and 58 . Similar results were achieved with the back-projection technique (15b) and a desk calculator, but $r^{2}$ values were lower.

$$
\begin{aligned}
& C_{p}^{\hat{\text { i.v. }}}=45.264 e^{-0.2512 t}+43.632 e^{-4.655 t} \\
& C_{p}^{\hat{p . o .}}=47.759 e^{-0.2501 t}+62.123 e^{-3.028 t}-322.2 e^{-7.362 t}+213.7 e^{-10.08 t}
\end{aligned}
$$

The $r^{2}$ value for the fit shown as equation 57 was 0.9997 and that for equation 58 was 1.000 . The area obtained from equation 57 was 189.56 $(99.8 \%$ of theoretical). The area obtained from equation 58 was 188.91 ( $100 \%$ of theoretical). Thus, in this case, the last two exponential terms of the polyexponential equation giving $C_{p}^{\text {i.v. }}$ as a function of time vanished. It is interesting in this example that at $7.5 \mathrm{~min}(0.125 \mathrm{hr})$ the third and fourth 
terms of equation 55 contribute 8.25 and $9.40 \%$, respectively, of the $C_{p}^{\text {i.v. }}$ values, yet the terms appeared to vanish when data from 0.125 to $24 \mathrm{hr}$ were evaluated.

\section{Model I-C}

Part A

For model I-C, the $\lambda_{i}$ 's are the absolute values of the negative roots of the cubic equation 59, where $s$ is the Laplace variable introduced.

$$
s^{3}+a_{2} s^{2}+a_{1} s+a_{0}=0
$$

where

$a_{2}=E_{1}+E_{2}+E_{3}$

$a_{1}=E_{1} E_{2}+E_{1} E_{3}+E_{2} E_{3}-k_{23} k_{32}-k_{12} k_{21}-k_{13} k_{31}$

$a_{0}=E_{1} E_{2} E_{3}-k_{23} k_{32} E_{1}-k_{12} k_{21} E_{3}-k_{13} k_{21} k_{32}-k_{12} k_{23} k_{31}-k_{13} k_{31} E_{2}(62)$

Simulations were performed with equations $59-62$ by letting $k_{13}=$ $0.25, k_{31}=0.9, k_{21}=0, k_{30}=0.1, k_{23}=4, k_{32}=0.5, E_{2}=k_{23}=4$, and $E_{3}=$ 1.5 and by varying $k_{12}$. Values of $k_{12}=1.00,9.75$, and 14.75 were used, making $E_{1}=1.25,10$, and 15 , respectively. Results are shown in Table III. These results support the statement that when everything else remains constant, and $k_{12}$ (and $E_{1}$ ) increases, the absolute value of the difference between $E_{1}$ and $\lambda_{3}$ becomes smaller and smaller. The opposite trend occurs with the difference between $E_{1}$ and $\lambda_{2}$. Both effects can cause collapsing.

\section{Part B}

A simulation was performed by letting $C_{0}=C_{0}^{\prime}=100, k_{13}=1.67 \mathrm{hr}^{-1}$, $\mathrm{k}_{31}=0.0145 \mathrm{hr}^{-1}, k_{23}=3.5 \mathrm{hr}^{-1}, k_{32}=0.455 \mathrm{hr}^{-1}$, and $k_{30}=1.386 \mathrm{hr}^{-1}$. The reverse rat constants $k_{31}$ and $k_{32}$ are those based on Fick's law assuming volumes of 100,1500 , and $11,500 \mathrm{ml}$ for compartments 1,2 , and 3 , respectively. Substitution of the appropriate values into equations 59-62 and use of a cube root program and an electronic calculator gave eigenvalues

Table III. Results of Simulations with Equations 59-62 for Model I-C

\begin{tabular}{rcccccc}
\hline$k_{12}$ & $E_{1}$ & $\lambda_{1}$ & \multicolumn{1}{c}{$\lambda_{2}$} & \multicolumn{1}{c}{$\lambda_{3}$} & $\left|E_{1}-\lambda_{3}\right|$ & $\left|E_{1}-\lambda_{2}\right|$ \\
\hline 1.00 & 1.25 & $\mathbf{0 . 0 4 8 4}$ & 2.4009 & 4.3007 & 3.051 & 1.15 \\
9.75 & 10 & 0.0693 & 6.366 & 9.065 & 0.935 & 3.634 \\
14.75 & 15 & 0.0711 & 5.798 & 14.632 & 0.368 & 9.202 \\
\hline
\end{tabular}


of $1.1381,4.2278$, and 11.6596. Substitution into equations 19 and 22 gave equations 63 and 64 , respectively.

$$
\begin{aligned}
& C_{p}^{\text {i.v. }}=76.5199 e^{-1.1381 t}+23.5887 e^{-4.2278 t}-0.1086 e^{-11.6596 t} \\
& C_{p}^{\text {p.o. }}=119.7982 e^{-1.1381 t}-147.1322 e^{-4.2278 t}+27.3340 e^{-11.6596 t}
\end{aligned}
$$

Integration of equation 63 between the limits 0 and $\infty$ gave an area of 72.8049 ; the first, second, and third terms of equation 63 contribute 92.35 , 7.66 , and $-0.01 \%$, respectively, toward the total area. Thus the third term would readily vanish. This is also an interesting example in that it illustrates that an intravenous equation can have a negative term. Integration of equation 64 between the same limits gave the same area; the contributions of the first, second, and third terms of equation 64 toward the total area are $144.6,-47.8$, and $3.2 \%$, respectively. Thus the third term of the oral equation could also readily vanish. This example utilized volumes which are reasonably "physiological" for either unbound substances or free (unbound) drug existing in the body with plasma-protein- and tissue-bound drug. The rate constants utilized were also consistent with material covered in the Introduction.

In models I-B, I-C, II-B, and II-C, $k_{12}$ represents the rate constant for stomach emptying. This is known to be a rapid process, particularly in the fasting state and for small volumes of fluid, such as when medication is ingested. Hunt and Macdonald (30) reported an average half-life of $8.4 \mathrm{~min}$ for the stomach emptying of a $330-\mathrm{ml}$ pectin meal in ten subjects; this corresponds to a first-order rate constant of $4.95 \mathrm{hr}^{-1}$. The rate constants were larger for smaller volumes; extrapolation of their data suggests a rate constant of $9-13 \mathrm{hr}^{-1}$ for a volume of $150 \mathrm{ml}$. Eisner and Berger (31) reported an average half-life of $10.2 \mathrm{~min}$ for gastric emptying of $750 \mathrm{ml}$ of water in five subjects; this corresponds to a first-order rate constant of $4.08 \mathrm{hr}^{-1}$.

\section{DISCUSSION}

The relative magnitudes of the $E_{i}$ 's and $\lambda_{i}$ 's, coupled with the magnitude of $e^{-\lambda_{t} t}$ when $\lambda_{i}$ is large, is the cause of the vanishing exponential terms in the intravenous equations. Because of the form of the equations, this is unlikely to occur with the oral equations, but examples were shown in which vanishing could also occur in oral equations. As shown in the experimental section under model II-C, both the third and fourth terms of the intravenous, but not the oral, equation may vanish. This is not to say that exponential terms will always vanish in the intravenous equations, but rather that there is a high probability that this will or does occur. 
Generally accepted concepts in linear pharmacokinetics are the following: (a) the number of exponential terms required to fit the plasma concentration-time curve following intravenous administration is equal to the number of compartments in the disposition model, and the latter is independent of the input portion involved on oral administration, and (b) application of the Loo-Riegelman method to plasma cincentration data obtained following oral administration leads to estimation of intrinsic absorption rate constants. The models and the collapsing concept discussed in this report suggest that we must reassess these concepts.

The models discussed in this report involve transfer of drug back and forth between the "input" and "disposition" portions. These models relate more closely to known facts about drug absorption and disposition than the classical models. Since one and sometimes two of the exponential terms in the equation giving the plasma concentration as a function of time following intravenous administration are often extremely unimportant as contributors to either $C_{p}^{\mathrm{i} . v .}$ or the area under the curve, they appear to vanish when the data are evaluated by conventional methods. This has important implications as evidenced by the summary in Table IV. One can see from Table IV that now there is a great deal of ambiguity about which model to choose based on the number of exponential terms required to fit both intravenous and oral data in the practical sense. Even more disturbing is the fact that the observation of two exponential terms from intravenous data and three exponential terms from oral data now leads to 16 possibilities, and that some of these are actually "one-compartment open models" in the classical sense. Hence, with some drugs, the so-called $\alpha$-phase following intravenous administration may not be caused by tissue distribution at all but rather by recycling of drug back to the stomach and the intestinal contents by secreting processes. In most cases, the $\alpha$-phase is probably caused by both factors.

When the Wagner-Nelson method is applied to oral data obeying models I-A, I-B, and I-C and the Loo-Riegelman method is applied to oral data obeying models II-A, II-B, and II-C, conventional methods of resolving the resulting $A_{t} / V_{p}, t$ data provide estimates of the $\lambda_{i}$ 's and not of the absorption rate constants. These $\lambda_{i}$ 's are functions of all the rate constants in the model. The fundamental difference between the integrated expressions in this report and those of classical models is that the denominators of the coefficients and the exponents of the exponentials contain $\lambda_{i}$ values in the former and $k_{i j}$ values in the latter. The most important implication is that in most cases we really cannot distinguish one model from another based on the number of exponential terms. We have always realized that there are three two-compartment disposition models each of which yields a $C_{p}^{\text {i.v. }}$ equation 
Table IV. Summary of Potential Model Assignments

\begin{tabular}{|c|c|c|c|c|c|c|c|}
\hline \multicolumn{2}{|c|}{ Observations } & & & & & & \\
\hline \multirow{2}{*}{$\begin{array}{l}\text { Number of } \\
\text { exponential } \\
\text { terms } \\
\text { describing } \\
\text { intravenous } \\
\text { data }\end{array}$} & \multirow{2}{*}{$\begin{array}{c}\text { Number of } \\
\text { exponential } \\
\text { terms } \\
\text { describing } \\
\text { oral } \\
\text { data }\end{array}$} & \multicolumn{2}{|c|}{$\begin{array}{c}\text { Nonclassical } \\
\text { models }\end{array}$} & \multicolumn{2}{|c|}{ Classical models } & \multicolumn{2}{|l|}{ Totals $^{a}$} \\
\hline & & \multicolumn{2}{|c|}{$\begin{array}{l}\text { Basic Additional } \\
\text { model vaineties }\end{array}$} & Intravenous & Oral & Intravenous & Oral \\
\hline 1 & 2 & $I-A^{b}$ & 0 & 1 & 1 & 2 & 2 \\
\hline 2 & 2 & I-A & 0 & 0 & 0 & 1 & 1 \\
\hline 1 & 3 & $\left\{\begin{array}{c}\mathrm{I}-\mathrm{B}^{b} \\
\mathrm{I}-\mathrm{C}^{b} \\
\mathrm{II}-\mathrm{A}^{b}\end{array}\right.$ & $\left.\begin{array}{c}1^{b} \\
7^{b} \\
2^{b}\end{array}\right\}$ & 1 & 1 & 15 & 15 \\
\hline 2 & 3 & $\left\{\begin{array}{c}\mathrm{I}-\mathrm{B}^{b} \\
\mathbf{I}-\mathrm{C}^{b} \\
\mathrm{II}-\mathrm{A}^{b}\end{array}\right.$ & $\left.\begin{array}{l}1^{b} \\
7^{b} \\
2^{b}\end{array}\right\}$ & 3 & 3 & 16 & 16 \\
\hline 2 & 4 & II-B ${ }^{b}$ & $\left.\begin{array}{l}5^{b} \\
21^{b}\end{array}\right\}$ & 3 & 3 & 31 & 31 \\
\hline 3 & 3 & $\left\{\begin{array}{c}\mathrm{I}-\mathrm{B} \\
\mathrm{I}-\mathrm{C} \\
\mathrm{II}-\mathrm{A} \\
\mathrm{II}-\mathrm{B}^{b} \\
\mathrm{II}^{b} \mathrm{C}^{b}\end{array}\right.$ & $\left.\begin{array}{c}1 \\
7 \\
2 \\
5^{b} \\
21^{b}\end{array}\right\}$ & 21 & 0 & 62 & 41 \\
\hline 3 & 4 & $\left\{\begin{array}{l}\text { II-B }{ }^{b} \\
\text { II-C }\end{array}\right.$ & $\left.\begin{array}{r}5^{b} \\
21^{b}\end{array}\right\}$ & 21 & 21 & 49 & 49 \\
\hline 4 & 4 & $\left\{\begin{array}{l}\text { II-B } \\
\text { II-C }\end{array}\right.$ & $\left.\begin{array}{r}5 \\
21\end{array}\right\}$ & 90 & 0 & 118 & 28 \\
\hline
\end{tabular}

${ }^{a}$ This number excludes other types of collapsing such as discussed by Ronfeld and Benet (37). ${ }^{b}$ Collapsed equations following intravenous administration.

with two exponential terms. However, Table IV indicates that there are now 16 potential models which can explain two exponential terms following bolus intravenous injection or post-constant-rate infusion. This presents a real problem to the pharmacokineticist.

The "answer" to the problem from the author's viewpoint is to concentrate in the future on model-independent linear pharmacokinetics. The first step in the analysis of a given set of linear data with this approach is to find 
initial estimates of the $I_{i}$ and $\lambda_{i}$ values for the general polyexponential equation shown as equation 65 .

$$
C_{p}=\sum_{i=1}^{n} I_{i} e^{-\lambda_{i} t}
$$

Now the $\lambda_{i}$ 's may or may not be $k_{i j}$ 's. The approach suggests that it may be very difficult to determine whether they are $\lambda_{i}$ 's or $k_{i j}$ 's in a given case and the search may not be worthwhile and may be put aside. Fortunately, the "stripping" or back-projection technique has now been automated and is contained in the program AUTOAN (32) and in a separate program, CSTRIP (38). However, I must caution the reader that CSTRIP (which also obtains the initial estimates in AUTOAN 1 and AUTOAN 2) does not provide least-squares estimates of the $I_{i}$ 's and $\lambda_{i}$ 's of equation 65 . It just does what we have been doing with a desk calculator, only it usually does the job better. Such parameter estimates should only be viewed as initial estimates except in cases where there is virtually no error in the data and the $r^{2}$ value is very close to unity. These initial estimates may then be used with a program such as NONLIN (29) to obtain final least-squares estimates of the $I_{i}$ and $\lambda_{i}$ values. Usually the more error in the data the greater will be the improvement in the fit from CSTRIP or calculator to nonlinear least squares. Alternatively, AUTOAN1 or AUTOAN 2 will choose a specific model, use the initial estimates as starting values, and then obtain a least-squares fit to the particular model it tells one that it chose. One can then substitute the least-squares parameter estimates into the $C_{p}, t$ equation for that model and produce a numerical polyexponential equation which fits the data very well (in most cases). Thus, although the author recommended otherwise in a recent article (33), he has now, in light of the evidence presented in this article, changed his mind and recommends simple least-squares polyexponential fitting as previously recommended by Koizumi et al. (34) and Nüesch (35).

The usually desired parameters, such as half-lives, $F, F^{*}, V_{p}$, timedependent volume of distribution (36), volumes equivalent to $V_{d \text { area }}$ or $V_{\beta}$, and sometimes even absorption rate constants, may then be directly calculated from the numerical polyexponential equation. One can also predict multiple-dose blood levels and perform essentially all needed dosage regimen calculations as has been shown (15c). A careful review by the pharmacokineticist will reveal that in most cases it is not necessary to derive the values of microscopic rate constants at all. In fact, this practice makes more work than necessary. At first glance, this may appear to take some of the glamor out of pharmacokinetics. On the contrary, the author believes it presents a challenge to see what really can be done with model-independent 
pharmacokinetics. A previous article (27) was an initial effort and a future one in this journal will yield additional useful equations.

\section{APPENDIX: COMPARISON OF OBSERVED AND ESTIMATED VALUES FOR EXAMPLES}

\begin{tabular}{|c|c|c|c|c|c|}
\hline \multicolumn{6}{|c|}{ Model II-A (Example 1) } \\
\hline$C_{p}^{\text {i.v. }}$ & $\hat{C_{p}^{\text {i.v. }}}$ & $\%$ deviation & $C_{p}^{\text {p.o. }}$ & $\hat{C_{p}^{p . o .}}$ & $\%$ deviation \\
\hline 80.06 & 79.74 & 0.43 & 33.21 & 34.10 & -2.7 \\
\hline 65.24 & 65.34 & -0.15 & 47.62 & 48.57 & -2.0 \\
\hline 45.67 & 45.83 & -0.36 & 50.94 & 51.41 & -0.93 \\
\hline 34.25 & 34.27 & -0.06 & 43.43 & 43.53 & -0.22 \\
\hline 27.38 & 27.34 & 0.13 & 35.27 & 35.20 & 0.19 \\
\hline 17.40 & 17.59 & -1.1 & 19.23 & 19.19 & 0.22 \\
\hline 13.23 & 13.52 & -2.2 & 13.62 & 13.62 & 0.00 \\
\hline 10.81 & 11.02 & -1.9 & 11.11 & 11.11 & 0.00 \\
\hline 8.85 & 8.99 & -1.5 & 9.096 & 9.096 & 0.00 \\
\hline 5.93 & 5.98 & -0.81 & 6.097 & 6.097 & 0.00 \\
\hline 1.79 & 1.76 & 1.3 & 1.836 & 1.836 & 0.00 \\
\hline
\end{tabular}

Model II-C

\begin{tabular}{|c|c|c|c|c|c|}
\hline$C_{p}^{i . v .}$ & $C_{p}^{\hat{i . v .}}$ & $\%$ deviation & $C_{p}^{p . o .}$ & $C_{p}^{p . o .}$ & $\%$ deviation \\
\hline 69.08 & 68.25 & 1.2 & 21.07 & 21.07 & 0.00 \\
\hline 55.47 & 56.14 & -1.2 & 40.05 & 40.05 & 0.00 \\
\hline 44.25 & 44.18 & 0.16 & 49.08 & 49.08 & 0.00 \\
\hline 39.07 & 38.82 & 0.64 & 44.83 & 44.83 & 0.00 \\
\hline 35.67 & 35.62 & 0.13 & 40.00 & 40.01 & -0.025 \\
\hline 30.82 & 31.09 & -0.89 & 33.48 & 33.48 & 0.00 \\
\hline 27.05 & 27.39 & -1.3 & 29.11 & 29.11 & 0.00 \\
\hline 21.03 & 21.30 & -1.3 & 22.56 & 22.56 & 0.00 \\
\hline 16.38 & 16.57 & -1.2 & 17.56 & 17.57 & -0.06 \\
\hline 9.933 & 10.03 & -0.95 & 10.65 & 10.65 & 0.00 \\
\hline 6.025 & 6.067 & -0.70 & 6.461 & 6.460 & 0.015 \\
\hline 2.216 & 2.221 & -0.24 & 2.377 & 2.376 & 0.042 \\
\hline 0.110 & 0.109 & 0.91 & 0.118 & 0.118 & 0.00 \\
\hline
\end{tabular}

\section{REFERENCES}

1. L. Z. Benet. General treatment of linear mammillary models with elimination from any compartment as used in pharmacokinetics. J. Pharm. Sci. 61:536-541 (1972).

2. D. P. Vaughan and A. Trainor. Derivation of general equations for linear mammillary models when drug is administered by different routes. J. Pharmacokin. Biopharm. 3:203218 (1975).

3. T. J. Benya and J. G. Wagner. Rapid equilibration of warfarin between tissue and plasma. J. Pharmacokin. Biopharm. 3:237-255 (1975).

4. P. A. Shore, B. B. Brodie, and C. A. M. Hogben. The gastric secretion of drugs. A $p$ H-partition hypothesis. J. Pharmacol. Exp. Ther. 119:361-369 (1957). 
5. C. A. M. Hogben, L. S. Schanker, D. J. Tocco, and B. B. Brodie. Absorption of drugs from the stomach. II. The human. J. Pharmacol. Exp. Ther. 120:540-545 (1957).

6. A. R. Cooke and A. Birchall. Absorption of ethanol from the stomach. Gastroenterology 57:269-272 (1969).

7. K. Hellström, A. Rosen, and Å. Swahn. Absorption and decomposition of potassium ${ }^{35}$ S-phenoxymethyl penicillin. Clin. Pharmacol. Ther. 16:826-833 (1974).

8. J. N. Hunt. The simultaneous estimation of the absorption of water and sulphaguanidine from the stomach of man. J. Physiol. (London) 109:134-141 (1949).

9. W. H. Hall and J. E. Doherty. Gastric digoxin absorption. Clin. Res. 19:33 (1971) (abst.).

10. T. Koisumi, T. Arita, and K. Kakemi. Absorption and excretion of drugs. XIX. Some pharmaceutic aspects of absorption and excretion of sulfonamides (1). Absorption from rat stomach. Chem. Pharm. Bull. (Tokyo) 12:413-420 (1964).

11. K. Kakemi, T. Arita, R. Hori, and R. Konishi. Absorption and excretion of drugs. XXX. Absorption of barbituric acid derivatives from the rat stomach. Chem. Pharm. Bull. (Tokyo) 15:1534-1539 (1967).

12. F. von Lauterbach. Resorption and Sekretion von Arzneistaffen durch die Mukosaepithelien des Gastrointestinaltraktes. In Transfermechanismen, 8th Deidensheimer Gespräch, May 11-12, 1974, pp. 63-89; also Arzneimittel-Forschung 25:459510 (1975).

13. W. G. Crouthamel and C. R. Abolin. Tetracycline absorption in the rat. Abstracts of papers presented at the 17th national meeting of the APhA Academy of Pharmaceutical Sciences, New Orleans, La., Vol. 4, No. 2, p. 121, 1974, abst. 10.

14. J. G. Wagner. Biopharmaceutics and Relevant Pharmacokinetics, Drug Intelligence Publications, Hamilton, Ill., 1975, (a) pp. 45-46, (b) p. 371, (c) p. 261.

15. J. G. Wagner. Fundamentals of Clinical Pharmacokinetics, Drug Intelligence Publications, Hamilton, Ill., 1975, (a) p. 34, (b) pp. 60, 80, 89, (c) pp. 129-172.

16. K. Kakemi, T. Arita, R. Hori, and R. Konishi. Absorption and excretion of drugs. XXXII. Absorption of barbituric acid derivatives from rat small intestine. Chem. Pharm. Bull (Tokyo) 15:1883-1887 (1967).

17. G. Fiese and J. H. Perrin. A note on the absorption of 3-methoxy- $N$-methyl morphinan hydrochloride from the rat stomach. J. Pharm. Pharmacol. 20:98-101 (1968).

18. G. Fiese and J. H. Perrin. Further investigations into the absorption of dextromethorphan from the rat's stomach. J. Pharm Sci. 58:599-601 (1969).

19. B. K. Martin. Accumulation of drug anions in gastric mucosal cells. Nature 198:896-897 (1963).

20. D. C. Taylor and R. U. Grundy. An investigation of the rat in situ absorption model using practolol and propranolol. J. Pharm. Pharmacol. Suppl. 27:65P (1975).

21. J. S. Fordtran and J. M. Dietschy. Water and electrolyte movement in the intestine. Gastroenterology 50:263-285 (1966).

22. H. W. Davenport. Physiology of the Digestive Tract: An Introductory Text, Yearbook Medical Publishers, Chicago, 1961, (a) pp. 162-176, (b) p. 75.

23. D. Winne. Formal kinetics of water and solute absorption with regard to intestinal blood flow. J. Theor. Biol. 27:1-18 (1970).

24. J. H. Williams, Jr., M. Mager, and E. D. Jacobson. Relationship of mesenteric blood flow to intestinal absorption of carbohydrates. J. Lab. Clin. Med. 63:853-863 (1964).

25. D. R. Holland and J. F. Quay. Intestinal secretion of erythromycin base. J. Pharm. Sci. 65:417-419 (1976).

26. J. C. K. Loo and S. Riegelman. New method for calculating the intrinsic absorption rate of drugs. J. Pharm. Sci. 57:918-928 (1968).

27. J. G. Wagner. Do you need a pharmacokinetic model, and, if so, which one? J. Pharmacokin. Biopharm. 3:457-478 (1975).

28. J. G. Wagner and E. Nelson. Percent absorbed time plots from blood level and/or urinary excretion data. J. Pharm. Sci. 52:610-611 (1963).

29. C. M. Metzler. Technical Report 7292/69/7292/005, Upjohn, November 25, 1969.

30. J. N. Hunt and I. Macdonald. The influence of volume on gastric emptying. J. Physiol. (London) 126:459-474 (1954). 
31. M. Eisner and W. Berger. Biguanides and gastric emptying in man. Digestion 4:309-313 (1971).

32. A. J. Sedman and J. G. Wagner. AUTOAN, A Decision-Making Pharmacokinetic Computer Program, distributed by Publication Distribution Service, 615 E. University Ave., Ann Arbor, Mich. 48109, November 11, 1974, 102 pp.

33. J. G. Wagner. Pharmacokinetics and bioavailability. Triangle 14:101-108 (1975).

34. T. Koizumi, M. Ueda, and K. Kakemi. The routine fitting of pharmacokinetic data to multiexponential equation. Chem. Pharm. Bull. (Tokyo) 21:2549-2556 (1973).

35. E. Nüesch. Proof of the general validity of Dost's law of corresponding areas. Eur.J. Clin. Pharmacol. 6:33-43 (1973).

36. S. Niazi. Volume of distribution as a function of time. J. Pharm. Sci. 65:452-454 (1976).

37. R. A. Ronfeld and L. Z. Benet. Interpretation of plasma concentration time curves after oral dosing. J. Pharm. Sci. (in press, 1976).

38. A. J. Sedman and J. G. Wagner. CSTRIP, a fortran IV computer program for obtaining initial polyexponential parameter estimates. $J$. Pharm. Sci. 65:1006-1010 (1976). 